\title{
Frequency spectra of cosmic ray air shower radio emission measured with LOPES
}

\author{
A. Nigl ${ }^{1}$, W.D. Apel ${ }^{2}$, J.C. Arteaga ${ }^{3}$, T. Asch ${ }^{4}$, J. Auffenberg ${ }^{5}$, F. Badea ${ }^{2}$, L. Bähren ${ }^{6}$, K. Bekk ${ }^{2}$, \\ M. Bertaina ${ }^{7}$, P.L. Biermann ${ }^{8}$, J. Blümer ${ }^{2,3}$, H. Bozdog ${ }^{2}$, I.M. Brancus ${ }^{9}$, M. Brüggemann ${ }^{10}$, P. Buchholz ${ }^{10}$, \\ S. Buitink ${ }^{1}$, H. Butcher ${ }^{6}$, E. Cantoni ${ }^{7}$, A. Chiavassa ${ }^{7}$, F. Cossavella ${ }^{3}$, K. Daumiller ${ }^{2}$, V. de Souza ${ }^{3}$, F. Di \\ Pierro $^{7}$, P. Doll ${ }^{2}$, R. Engel ${ }^{2}$, H. Falcke ${ }^{1,6}$, H. Gemmeke ${ }^{4}$, P.L. Ghia ${ }^{11}$, R. Glasstetter ${ }^{5}$, C. Grupen ${ }^{10}$, \\ A. Haungs ${ }^{2}$, D. Heck ${ }^{2}$, J.R. Hörandel ${ }^{1}$, A. Horneffer ${ }^{1}$, T. Huege ${ }^{2}$, P.G. Isar ${ }^{2}$, K.-H. Kampert ${ }^{5}$, \\ D. Kickelbick ${ }^{10}$, Y. Kolotaev ${ }^{10}$, O. Krömer ${ }^{4}$, J. Kuijpers ${ }^{1}$, S. Lafebre ${ }^{1}$, P. Łuczak $^{12}$, M. Manewald ${ }^{4}$, \\ H.J. Mathes ${ }^{2}$, H.J. Mayer ${ }^{2}$, C. Meurer ${ }^{2}$, B. Mitrica ${ }^{9}$, C. Morello ${ }^{11}$, G. Navarra ${ }^{7}$, S. Nehls ${ }^{2}$, J. Oehlschläger ${ }^{2}$, \\ S. Ostapchenko ${ }^{2}$, S. Over ${ }^{10}$, M. Petcu ${ }^{9}$, T. Pierog ${ }^{2}$, J. Rautenberg ${ }^{5}$, H. Rebel ${ }^{2}$, M. Roth ${ }^{2}$, A. Saftoiu ${ }^{9}$, \\ H. Schieler ${ }^{2}$, A. Schmidt ${ }^{4}$, F. Schröder ${ }^{2}$, O. Sima $^{13}$, K. Singh ${ }^{1}$, M. Stümpert ${ }^{3}$, G. Toma ${ }^{9}$, G.C. Trinchero ${ }^{11}$, \\ H. Ulrich ${ }^{2}$, J. van Buren ${ }^{2}$, W. Walkowiak ${ }^{10}$, A. Weindl ${ }^{2}$, J. Wochele ${ }^{2}$, J. Zabierowski ${ }^{12}$, and J.A. Zensus ${ }^{8}$ \\ (Affiliations can be found after the references)
}

Received 7 December 2008 / Accepted 17 June 2008

\begin{abstract}
Aims. We wish to study the spectral dependence of the radio emission from cosmic-ray air showers around $100 \mathrm{PeV}\left(10^{17} \mathrm{eV}\right)$. Methods. We observe short radio pulses in a broad frequency band with the dipole-interferometer LOPES (LOFAR Prototype Station), which is triggered by a particle detector array named Karlsruhe Shower Core and Array Detector (KASCADE). LOFAR ' is the Low Frequency Array. For this analysis, 23 strong air shower events are selected using parameters from KASCADE. The ' radio data are digitally beam-formed before the spectra are determined by sub-band filtering and fast Fourier transformation.

Results. The resulting electric field spectra fall off to higher frequencies. An average electric field spectrum is fitted with an ' exponential $E_{\nu}=K \cdot \exp (\nu / \mathrm{MHz} / \beta)$ and $\beta=-0.017 \pm 0.004$, or alternatively, with a power law $\epsilon_{\nu}=K \cdot \nu^{\alpha}$ and a spectral index of $\alpha=-1 \pm 0.2$. The spectral slope obtained is not consistent within uncertainties and it is slightly steeper than the slope obtained from Monte Carlo simulations based on air showers simulated with CORSIKA (Cosmic Ray Simulations for KASCADE). For the analyzed sample of LOPES events, we do not find any significant dependence of the spectral slope on the electric field amplitude, the azimuth angle, the zenith angle, the curvature radius, nor on the average distance of the antennae from the shower ' core position. But one of the strongest events was measured during thunderstorm activity in the vicinity of LOPES and shows the longest pulse length measured of $110 \mathrm{~ns}$ and a spectral slope of $\alpha=-3.6$.

Conclusions. We show with two different methods that frequency spectra from air shower radio emission can be reconstructed on - event-by-event basis, with only two dozen dipole antennae simultaneously over a broad range of frequencies. According to the ' obtained spectral slopes, the maximum power is emitted below $40 \mathrm{MHz}$. Furthermore, the decrease in power to higher frequencies ' indicates a loss in coherence determined by the shower disc thickness. We conclude that a broader bandwidth, larger collecting area, and longer baselines, as will be provided by LOFAR, are necessary to further investigate the relation of the coherence, pulse length, and spectral slope of cosmic ray air showers.
\end{abstract}

Key words. acceleration of particles - elementary particles - radiation mechanisms: non-thermal - instrumentation: detectors methods: data analysis

\section{Introduction}

Cosmic rays are particles or nuclei constantly bombarding the Earth's atmosphere with a large spread in energy. The origin of cosmic rays with the highest energies is still unknown and therefore subject of intensive research (Auger Collaboration: J. Abraham et al. 2007). These particles, mostly protons, initiate an extensive shower of secondary particles traveling with almost the speed of light through the air. The charged particles in the air shower produce electromagnetic emission relativistically beamed in the forward direction. This work probes the spectral dependence of the electromagnetic field produced in these

Send offprint requests to: A. Nigl

Correspondence to: anigl@astro.ru.nl air showers to investigate the radio emission mechanism. Understanding the emission mechanism and its dependence on frequency and distance to the shower core will be an important step to infer the primary particle species of the cosmic ray.

The radio emission of charged particles in air showers was first observed in 1964 by Jelley et al. (1965) at $44 \mathrm{MHz}$ and by Allan \& Jones 1966 at $60 \mathrm{MHz}$. Those early studies found strongly pulsed radio emission from air showers detected with radio antennae triggered by particle detectors. A dependence of the radio emission's polarization on the geomagnetic field favored a geomagnetic emission mechanism (see, e.g., Allan et al. 1967, 1969; Sun 1975). The first quantitative radio frequency spectra were obtained from a few simultaneous narrowband observations below 
Nigl et al.: CR electric field spectrum with LOPES

$100 \mathrm{MHz}$ by, e.g., Spencer (1969), Allan et al. (1970), and Prah (1971). In this earlier work, spectra decreasing in frequency were obtained with spectral indices $\alpha$ between -2 and -1 . However, radio measurements were abandoned in the late 1970s because of strong radio frequency interference in the signal, difficulties with the interpretation of the measurements, and because of the success of alternative observing techniques. An excellent review of the early work can be found in Allan (1971).

Interest in the radio technique was revived by the LOFAR Prototype Station (LOPES) group Falcke et al. 2005) and by the Cosmic ray Detection Array with Logarithmic ElectroMagnetic Antennas (CODALEMA) group (Ardouin et al. 2006), both using digital data acquisition and powerful computers for data analysis on digitized radio signal for the first time. LOFAR is the Low Frequency Array. LOPES measured a linear dependence of the electric field amplitude of the radio emission in extensive cosmicray air showers on the energy of the primary particle. This result was an important step in proving the radio emission process to be coherent.

The following two radio emission mechanisms are believed to mainly contribute to the production of electromagnetic radiation in air showers. First, the geomagnetic mechanism generates beamed synchrotron emission through relativistic electron-positron pairs in the Earth's magnetic field, which is believed to be the dominant mechanism (Allan et al. 1967; Allan et al. 1969). This ansatz led to the description of coherent geosynchrotron emission at meter wavelengths (Falcke \& Gorham 2003). Second, relativistic particles emit Cherenkov radiation as a kind of photonic shockwave (Askaryan 1962, 1965; Kahn \& Lerche 1966). However, the Cherenkov emission mechanism is more efficient in denser media than air, like water and ice. Additionally, radio emission in air showers was claimed to be dependent on the geoelectric field, and it was found that strong electric fields in thunderstorm clouds can amplify the measured radio amplitudes (Mandolesi et al. 1974; Gurevich et al. 2004; Buitink et al. 2007).

When a cosmic ray initiates an extensive air shower, a thin shower pancake of particles is formed growing to about a few hundred meters in diameter. The pancake has a thickness of a few meters, due to particle attenuation in the atmosphere and relativistic beaming at high gamma factors. Close to the axis of the cosmic-ray air shower, the particle pancake is thinnest and thus the pulse profile received on the axis is expected to be shortest. The radio pulse is expected to broaden with distance from the shower core, mainly caused by geometric-delays in the arrival time of longitudinal shower evolution stages (Huege et al. 2007). The spectral shape of the radio emission is mainly determined by the distribution of electrons and positrons in the shower pancake, as they emit synchrotron radiation. According to Monte Carlo simulations, the spectrum starts with coherent emission at wavelengths larger than the shower pancake thickness, and it falls off exponentially to higher frequencies, where coherence will be lost when the wavelength becomes comparable with the shower thickness (Huege \& Falcke 2005a).

A cosmic-ray radio spectrum is particularly interesting to probe the coherence of the geosynchrotron emission and to study the width of the radio pancake, which determines the length of the detected pulse. This is important for future instruments, such as LOFAR, which will provide even larger bandwidths and baselines than LOPES.

We present electric field spectra determined from data recorded by LOPES with 30 antennae dipoles. The instruments LOPES and KASCADE are described in Sect. 2, The selection and data analysis of radio events are explained in Sect. 3 and in Sect. 4 respectively. The obtained spectra are shown and discussed in Sect. 5.

\section{Instrument}

The LOFAR prototype station LOPES (www.lopes-project.org, Horneffer et al. 2004; Falcke et al. 2005) has been optimized for the detection of cosmic rays with energies around $10^{17} \mathrm{eV}$. LOPES is placed in between particle detectors of the experiment KASCADE (www-ik.fzk.de/KASCADE_home.html, Antoni et al. 2003) in Karlsruhe. The data for this work were taken with LOPES operating 30 early LOFAR prototype dipole antennae. KASCADE is the Karlsruhe Shower Core and Array Detector located at the Forschungszentrum Karlsruhe, Germany. It is made up of $16 \times 16$ particle detector stations placed on a $200 \times 200 \mathrm{~m}^{2}$ rectangular grid, with $13 \mathrm{~m}$ spacing, a central hadron calorimeter, and a muon tracking detector. A layout of LOPES and KASCADE on the site of the Forschungszentrum Karlsruhe is shown in Fig. 1

KASCADE provides LOPES with a cosmic-ray trigger as well as particle shower parameters, like muon number, electron number, direction of origin, and position of the shower center (Antoni et al. 2003). These parameters have been used to select cosmic-ray events for LOPES postprocessing.

The LOPES hardware digitizes the radio signals measured by the dipole antenna wires with a rate of 80 Megasamples per second and in a dynamic range of 12 bits. The frequency band used for cosmic-ray detection ranges from $43 \mathrm{MHz}$ to $74 \mathrm{MHz}$, since the bandpass filter attenuates the received signal significantly outside these limits. The data is stored on custom-made PC boards with a $1 \mathrm{~GB}$ ring buffer for each dipole. The readout time of a $0.8 \mathrm{~ms}$ cosmic-ray event takes about $1.5 \mathrm{~s}$ and the system is not sensitive to a new trigger for that period. The data taken with LOPES are matched with the KASCADE data and sent to storage units for further off-line analysis. LOPES was triggered with a large-event-trigger from KASCADE, which requires a detection in 10 out of the 16 detector clusters of KASCADE.

\subsection{Radio pulse broadening}

LOPES measures the pulse at antenna level, thus at different positions and distances from the shower core at the same time. Radio frequency interference (RFI) is rejected by down-weighting of narrow spectral lines that lie three sigmas above the average spectrum in two iterations. The signals of all antenna dipoles are combined by beamforming to increase sensitivity and to further suppress RFI. The 30 antenna positions in the LOPES layout result in an average distance of the antennae from any possible shower core position within KASCADE of $\bar{d}=75 \mathrm{~m}$.

Furthermore, the signal detected by the LOPES dipoles is bandpass filtered within the limits from $40 \mathrm{MHz}$ to 


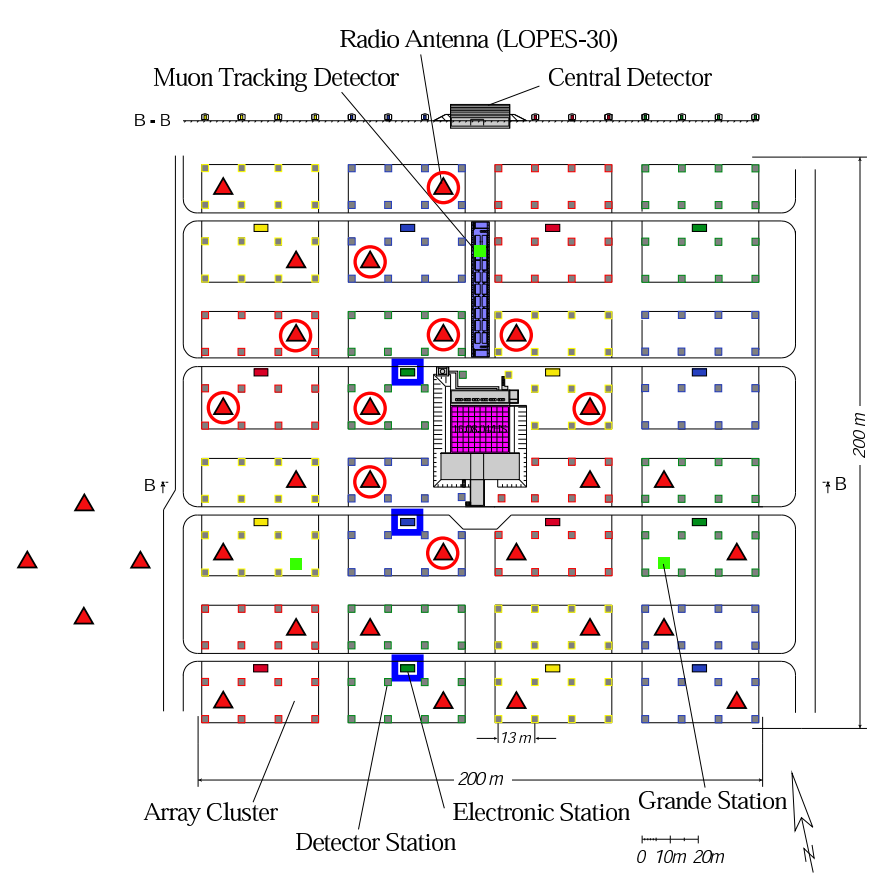

Figure 1. Layout of LOPES inside the KASCADE array (Horneffer 2006). The 252 small squares indicate the KASCADE particle detectors. The triangles show the positions of the LOPES antennae, as they were positioned for this work, and the circles highlight the first stage of LOPES with 10 operational antennae. The three rectangles mark the electronic stations that house the LOPES electronics. Each of these stations is collecting data from ten antennae elements. The top station holds the master clock module and a board for the reception of the KASCADE timestamp. Among the KASCADE detectors are three KASCADEGrande (Navarra et al. 2004) detectors (squares), which are spaced on a bigger grid to cover a larger area and thus higher primary particle energies.

$80 \mathrm{MHz}$. In principle, the filtered bandwidth of $40 \mathrm{MHz}$ broadens an infinitely short pulse in time to $25 \mathrm{~ns}(\Delta t \sim$ $1 / \Delta \nu)$ and changes the spectral slope outside the band due to attenuation. The impulse response of a first LOPES antenna fitted with a Gaussian resulted in a width of $57 \mathrm{~ns}$ (Horneffer 2006). Thus, additional broadening must be caused by the phase-characteristic of the LOPES hardware, but the spectral slope is not changed within the LOPES band. Therefore, the measured pulse lengths are upper limits and the minimum detectable width is close to $25 \mathrm{~ns}$.

\subsection{Gain calibration}

The inverted V-shaped dipole antennae of LOPES have a direction dependent antenna gain and the data acquisition hardware as a whole has a frequency dependent electronics gain. The electronics gain was determined for each antenna in several dedicated calibration campaigns by measuring a well-defined signal from a reference antenna mounted on a crane overhead LOPES (Nehls et al. 2007. subm., Fig. 2).

The direction and frequency dependent antenna gain calibration values $G_{(\phi, \theta, \nu)}$ for a LOPES antenna element, mounted on a metal pedestal, were calculated with a simulation model by Arts (2005). Here, $\phi$ is the azimuth angle

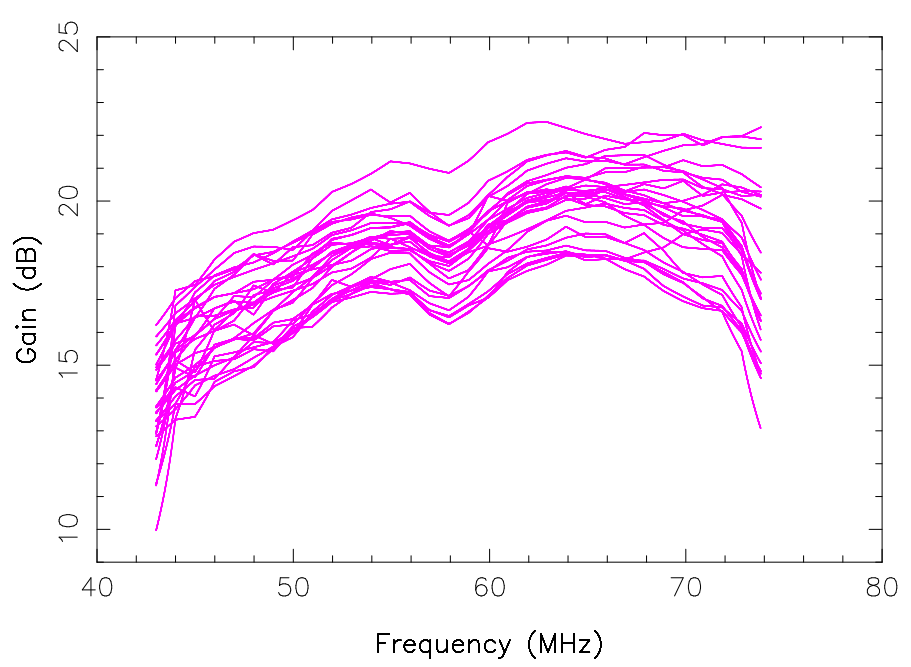

Figure 2. Electronics gain factors are plotted for all 30 LOPES antennae. The factors are for the correction of the received voltage measured in several calibration campaigns at KASCADE in the band from $43 \mathrm{MHz}$ to $74 \mathrm{MHz}$.

and $\theta$ is the elevation angle. The pedestal and the geometry of the LOPES dipoles make the gain pattern frequency dependent. At the lower end of the LOPES band, the contourlines of equal gain in the pattern are oval-shaped and the dipoles have their maximum sensitivity to the zenith with the major axis of the ellipse being perpendicular to the dipole, lying in East-West direction. With increasing frequency, the sensitivity maximum gets circular in shape concentrating around the zenith (in the middle of the band) and then the direction of maximum gain weakens, splits, and moves down in elevation at azimuth angles perpendicular to the dipole (see Fig. 31).

The output of the analog-digital converter (ADC) can be converted to units of field strength per unit bandwidth, using both sets of gains (Horneffer 2006):

$$
\begin{aligned}
\left\langle\varepsilon_{\nu}\right\rangle & =\frac{|\mathbf{E}|}{\Delta \nu} \\
& =\frac{1}{\Delta \nu} \sqrt{\frac{4 \pi \nu^{2} \mu_{0}}{G_{(\phi, \theta, \nu)}} P_{a n t}} \\
& =\frac{1}{\Delta \nu} \sqrt{\frac{4 \pi \nu^{2} \mu_{0}}{G_{(\phi, \theta, \nu)}} K_{\nu} \frac{V_{A D C}^{2}}{R_{A D C}}} .
\end{aligned}
$$

Here, $\mathbf{E}$ is the electric field from the air shower measured at the antenna; $\Delta \nu$ is the filtered bandwidth of the signal; $\nu$ is the observing frequency; $G_{(\phi, \theta, \nu)}$ is the direction and frequency dependent gain of the antenna (Fig. 3); $P_{\text {ant }}$ is the power received by the antenna; $K_{\nu}$ is the frequency dependent correction factor for the electronics; as plotted in Fig. 2 2 for each antenna; $V_{A D C}$ is the voltage digitized by the ADC; $R_{A D C}$ is the input impedance of the ADC; $\mu_{0}$ is the electromagnetic permeability; and $c$ the speed of light.

For earlier LOPES results, the directional gain was averaged in frequency and applied to the beam-formed timeseries. For this work, it was applied in the frequency domain to each individual frequency bin (see Sect. 4.1). 
Nigl et al.: CR electric field spectrum with LOPES

\section{Event selection}

For this analysis, we studied events of LOPES using the 30 antennae setup from 2005-11-16 to 2006-11-30. In this period, LOPES was recording in a single polarization configuration, all dipoles were parallel and most sensitive to radiation from the East-West direction. About one million events were triggered and recorded within this period.

Events were pre-selected on parameters provided by KASCADE and by estimating the electric field strength:

$$
\begin{aligned}
\epsilon_{\mathrm{est}}= & (53 \pm 4.9)\left[\frac{\mu \mathrm{V}}{\mathrm{m} \mathrm{MHz}}\right] \\
& \times((1.09 \pm 0.017)-\cos \vartheta) \\
& \times \exp \left(\frac{-\mathrm{R}_{\mathrm{SA}}}{(221 \pm 62) \mathrm{m}}\right)\left(\frac{\mathrm{N}_{\mu}}{10^{6}}\right)^{(0.99 \pm 0.04)}
\end{aligned}
$$

This formula is obtained from fits to all LOPES events detected with the standard analysis software (Horneffer et al. 2007). Here, $N_{\mu}$ is the muon number at distances between $40 \mathrm{~m}$ and $200 \mathrm{~m}$, which is provided by KASCADE, $\vartheta$ is the geomagnetic angle (calculated using the shower direction reconstructed by KASCADE), and $R_{S A}$ is the average distance of the antennae from the shower axis.

We limited the distance of the shower core from the KASCADE center to $91 \mathrm{~m}$ to make sure that the shower core is inside the KASCADE array so that the shower reconstruction by KASCADE provides high accuracy. For this work, 71 radio events were pre-selected with a lower limit of $4 \mu \mathrm{V} \mathrm{m}^{-1} \mathrm{MHz}^{-1}$ on the electric field strength obtained with Eq. 2.

For the spectral analysis, 23 events of the 71 events were selected with a lower limit on the signal-to-noise ratio (SNR) of the E-field amplitude and the E-field root-meansquare (RMS) of 5 for each spectral bin. For these 23 events, a radio spectrum was generated with the two methods described in Sect. 4. The estimated primary energy for these processed radio events lies mainly in the range from $10^{17} \mathrm{eV}$ to $10^{18} \mathrm{eV}$.

\section{Data reduction}

The standard analysis software is a modular software package and it was developed for the data reduction of LOPES radio events. We use this software to pre-process the data for this work. The software allows us to view the data at any step in the desired sequence of processing (Horneffer 2006). After calibration, digital filtering, and beam-forming of the selected events, we performed the spectral analysis according to the following processing pipeline.

\subsection{Processing pipeline}

Two methods have been used to produce the electric field spectra. First, the determination of the electric field amplitude of the cosmic-ray radio pulse detected in the antennae cross-correlation beam (see Sect.4.5) was filtered in several adjacent frequency bins of the LOPES band from $43 \mathrm{MHz}$ to $74 \mathrm{MHz}$. Second, a Fourier transformation was applied to a few tens of samples around the full-band radio peak detected in the simple antennae beam (see Sect. 4.4).

The processing structure for the production of the cosmic-ray electric field spectra is shown in Fig. 4. The digitized signal is converted from ADC counts (01) to voltage (02). A Hanning window is applied to the time-series data (03) to reduce leakage in the successive Fast Fourier Transform (FFT) (04). In the frequency domain, weights for the frequency dependent electronics gain (06), weights for correcting delays introduced by the instrument hardware (07) and weights to reduce RFI (08) are applied to the frequency bins (05).

The resulting calibrated antenna signals (09) (as measured at the antenna feeds) are multiplied with another set of complex weights to phase them in the direction of the cosmic ray (10). The direction is provided by the KASCADE experiment and the standard analysis software attempts to refine it by fitting a maximum to the cosmic-ray pulse emission in a radio skymap (see Sect.4.3).

The phased antenna spectra (12) are corrected by the directional gain of the antennae (11) and they are split in 16 sub-bands of $2.5 \mathrm{MHz}$ applying modified Hanning window functions (13) as described in Sect. 4.2. After inverse Fourier transformation, the digitally-filtered and calibrated antenna time-series (14) are cross-correlated (15) to form the cc-beam, which is described in Sect. 4.5. For some events it became necessary to review the antenna selection and the beam forming result, and to reject antennae that do not contribute to a coherent pulse, which is indicated by the recursive arrow in Fig. 4 .

From the resulting cc-beam (16) the background noise is subtracted (see Sect. 4.7) and the amplitude of the electric field strength of the pulse (17) is determined by averaging 32 samples (400 ns) around the pulse to obtain the electric field spectrum.

For the second method, the phased full-band antenna spectra (12) are inverse Fourier transformed back to time. The resulting filtered and calibrated antenna time-series are averaged to form the simple f-beam (see Sect.4.4). The cc-beam was not used here since it calculates the absolute value of the electric field in the beam, whereas for the FFT-method, the full phase information is needed and thus the f-beam was used instead (see Sect. 4.4). The f-beam is also reduced by its background noise level and a modified Hanning window is applied. Subsequently, the pulse found in the f-beam is Fourier transformed back to the frequency domain to obtain the electric field spectrum. For the FFT, 32 samples (400 ns) around the air shower pulse (see Fig. 5) were chosen to minimize a contribution to the signal from the particle detectors and to obtain a spectral resolution of $2.5 \mathrm{MHz}$ matching the resolution of the first method. The values in the top left corner of the plot read: in the 1st line: the event number, the LOPES frequency window from $43 \mathrm{MHz}$ to $74 \mathrm{MHz}$, the estimated primary energy of $3.3 \times 10^{17} \mathrm{eV}$, the total electron number of $10^{6.7}$, the truncated muon number of $10^{5.7}$, and the geomagnetic angle of $71^{\circ}$; in the 2nd line: the electric field amplitude of the cc-beam (cc) of $15.4 \mu \mathrm{V} \mathrm{m}^{-1} \mathrm{MHz}^{-1}$, the fullband peak SNR of $45 \sigma$ and the root-mean-square (RMS) of $0.3 \mu \mathrm{V} \mathrm{m}^{-1} \mathrm{MHz}^{-1}$; and in the $3 \mathrm{rd}$ line: the electric field amplitude of the f-beam (f) of $15 \mu \mathrm{V} \mathrm{m}^{-1} \mathrm{MHz}^{-1}$, the fullband peak SNR of $70 \sigma$ and the RMS of $0.2 \mu \mathrm{V} \mathrm{m}{ }^{-1} \mathrm{MHz}^{-1}$. The RMS of both full-band beams was determined on $0.2 \mathrm{~ms}$ of data well before and after the air shower pulse (see Fig. 6). 


\subsection{Frequency window function}

For the selection of the frequency sub-bands, a filter function has been applied. The simplest window is a rectangular window. However, the sharp edges introduce leakage. For less sharp edges, a modified Hanning window is used. The Hanning function applies only to the beginning quarter and to the end quarter of the window. The Hanning window is basically a cosine-function and it has to be applied to the frequency band around the center frequency increased by half the period of the cosine of the Hanning window to preserve the integral of the rectangular window (see Fig. 7).

\subsection{Core position and beam direction}

The core position and the direction used for beam-forming is provided by the KASCADE experiment. The accuracy of the core position and shower direction of KASCADE for the selected showers of high energy are $1 \mathrm{~m}$ and $0.1^{\circ}$, respectively, valid up to a zenith angle of $42^{\circ}$ (Antoni et al. 2003). The KASCADE direction is optimized by finding the position of maximum emission in a $4 \mathrm{D}$ radio image produced with the LOPES data. The axes of the four dimensions are time, azimuth angle, elevation angle, and curvature radius of an assumed spherical wavefront. The separation from the KASCADE direction for the selected events increases slightly with zenith angle and ranges up to $2.9^{\circ}$ with an average of $(1.0 \pm 0.5)^{\circ}$ for zenith angles smaller than $75^{\circ}$, which is consistent with an earlier result of $(0.8 \pm 0.4)^{\circ}$ in Falcke et al. (2005), where only events with a maximum zenith angle of $60^{\circ}$ were analyzed (one sigma statistical uncertainties).

\subsection{Field strength beam}

The field strength beam (f-beam) is the average of multiple antennae signals being shifted by geometrical delays for the beam direction. The delayed antennae signals are added and then divided by the number of antennae:

$S_{f}(t)=\frac{1}{N} \sum_{i=1}^{N} f_{i}\left(t-\tau_{i}\right)$.

Here, $N$ is the number of antennae, $f$ is the single antenna field strength time-series, and $\tau$ the signal arrival time delay for the beam direction.

\subsection{Cross-correlation beam}

The cross-correlation beam (cc-beam) is defined as the real part of a standard cross-correlation of multiple antennae signals, shifted by one set of signal delays for a certain direction. The data from each unique antenna pair are multiplied with each other, the resulting values are averaged, and then the square root is taken while preserving the sign:

$S_{c c}(t)= \pm \sqrt{\left|\frac{1}{N_{\text {pairs }}} \sum_{i=1}^{N-1} \sum_{j>i}^{N} f_{i}\left(t-\tau_{i}\right) f_{j}\left(t-\tau_{j}\right)\right|}$.

Here, $N$ is the number of antennae, $N_{\text {pairs }}=N(N-$ $1) / 2$ is the number of unique pairs of antennae, $f$ is the single antenna field strength time-series, and $\tau$ the signal arrival time delay for the beam direction. The advantage of the cc-beam, compared to the simple field strength beam, is that only the coherent signal is preserved within the beam and RFI, which is only seen by a single antenna is more strongly suppressed, since auto-correlation terms are not taken into account.

\subsection{Power beam}

The power beam (p-beam) is the average of all antenna auto-correlations:

$S_{p}(t)=\sqrt{\frac{1}{N} \sum_{i=1}^{N} f_{i}^{2}(t)}$.

Here, $N$ is the number of antennae and $f$ is the single antenna field strength time-series. The power beam is sensitive to the total power received by all the antennae from all directions, independent of the coherence of the signal.

\subsection{Background noise}

The system noise in a LOPES beam toward the zenith was determined by averaging background spectra of 664 arbitrary chosen events recorded over a period of one year $(11 / 2005$ - 11/2006). For these background spectra, short pulses in time and narrowband signals in frequency were attenuated by down-weighting of single samples in time and single bins in frequency containing power exceeding one sigma above the average in two iterations. The result in units of electric field strength is plotted in Fig. 8 and in units of noise temperature is plotted in Fig. 9. The power in the frequency bins at $52.5 \mathrm{MHz}$ and $67.5 \mathrm{MHz}$ of the field spectrum contained strong variations, which suggests that not all RFI could be rejected before the calculation of the spectrum. The spectral field strength was obtained by averaging the result of the FFT applied to the filtered timeseries of each event and the noise temperature was calculated on the average spectral power of all events. The latter plot also shows the galactic noise interpolated with a model for $45 \mathrm{MHz}$ to $408 \mathrm{MHz}: T_{\nu}=32 \mathrm{~K} \times(\nu / 408 \mathrm{MHz})^{-2.5}$ (Falcke \& Gorham 2003). The galactic noise is lying only slightly below the system noise of LOPES. Furthermore, the power during two periods of 48 hours, separated by half a year, showed a modulation with $20 \%$ of the received signal in accordance with the galactic plane crossing the zenith. Thus, a LOPES beam is sensitive to the galactic noise, but still dominated by the system noise. The values outside the band from $45 \mathrm{MHz}$ to $72.5 \mathrm{MHz}$ are strongly attenuated by the bandpass filter.

We calculated the level of the background noise for the cc-beam and for the f-beam on an event-by-event basis on two blocks of $0.2 \mathrm{~ms}$ in the first and second half of each event. On average in time $n_{c c}=(-0.13 \pm$ 0.06) $\mu \mathrm{V} \mathrm{m}^{-1} \mathrm{MHz}^{-1}$ and $n_{f}=(0.6 \pm 0.2) \mu \mathrm{V} \mathrm{m}^{-1} \mathrm{MHz}^{-1}$ (one sigma statistical uncertainty).

The parts of the event files for the noise-calculation leave out the event edges, which are affected by the Hanning window. Also, they leave out the cosmic-ray pulse and possible emission from the KASCADE particle detectors.

In the LOPES data, the signal of the particle detectors is trailing the radio signal of the air shower, and the KASCADE trigger arrives about $1.8 \mu$ s later at the LOPES electronics than the radio pulse. The peak of the 
KASCADE emission arrives shortly after the real air shower pulse, between $1.7 \mu \mathrm{s}$ and $1.5 \mu \mathrm{s}$ before the trigger arrives at the LOPES electronics and it is less than $1 \mu$ s wide. The emission from the particle detectors is received by each antenna from several directions. Therefore, the detector noise does not add coherently and it is attenuated in the beamforming process.

The root-mean-square (RMS) of the background of the cc-beam was determined for each frequency bin on the same parts of each event as the noise level above. The RMS of the f-beam of each event was determined as an average of 50 spectra calculated on blocks of 32 samples, which were chosen away from the cosmic-ray pulse.

\subsection{Slope correction}

In Fig. 10, the slope of the 664 noise spectra recorded over the earlier mentioned period of one year is plotted as a function of zenith angle. The plot shows a steepening of the slopes of events with higher zenith angles. The variation of the sky noise is averaged out over the period of one year and it is not observed to change with zenith angle. A small contribution might come from remaining RFI increasing in signal strength to the horizon. However, the main contribution is introduced by deviation of the frequency dependent antenna gain calibration from the real antenna gain. This dependence affecting the slopes of the air shower spectra was corrected by dividing each spectra by a normalized correction spectrum:

$\hat{\epsilon}_{\nu}=\nu^{\alpha_{f}-\alpha_{r}} \overline{\nu^{\alpha_{f}-\alpha_{r}}}$.

Here, $\hat{\epsilon}_{\nu}$ is the electric field amplitude for the frequency bin $\nu, \alpha_{f}$ is the slope parameter for the measured spectrum obtained from a fit to the slopes in Fig. 10 of $-0.008 \times\left(90-\theta /{ }^{\circ}\right)-1.2$, and $\alpha_{r}$ is the slope of the reference spectrum in the zenith (Fig. 8) of -1.2 .

\subsection{Pulse amplitude}

The field strength amplitude of the frequency filtered ccbeam was determined by averaging it over a time window with the size of the inverse sub-band. As an example, a broadened pulse for a sub-band of $\sim 1.3 \mathrm{MHz}$ is plotted in Fig. 11, The values in the top left corner of the plot read: event number 13 , frequency sub-band from $48.1 \mathrm{MHz}$ to $49.4 \mathrm{MHz}$; E-field peak SNR of $27.1 \sigma$ of the frequency bin and the offset between the full-band peak maximum and the sub-band peak maximum of -20.4 ns. The dot lies at a higher field strength, since its frequency bin lies below the center frequency of the full-band beam of $60 \mathrm{MHz}$. The cc-beam shows correlation fringes for the center frequency of the sub-band. For comparison, the Gauss-fit on the smoothed full-band peak was broadened in width by the total number of frequency bins of 24 . The plotted pulse for a sub-band below the center frequency of the LOPES band is higher than the full-band pulse, which is consistent with the negative slope in the resulting spectrum.

\subsection{Uncertainty estimation}

The uncertainty of the determined electric field strength is affected by the direction and frequency dependent gain factors, phase uncertainties introduced by the electronics
Table 1. List of uncertainties on the determined electric field strengths.

\begin{tabular}{|c|c|c|c|}
\hline Method & RMS & Gain Uncert. & Phase Uncert. \\
\hline \hline cc-beam & $1.59(7.1 \%)$ & $0.35(1.56 \%)$ & $1.13(5 \%)$ \\
\hline f-beam & $0.78(3.8 \%)$ & $0.32(1.56 \%)$ & $1.01(5 \%)$ \\
\hline
\end{tabular}

These values are calculated from all 23 analyzed events in units of $\mu \mathrm{V} \mathrm{m}^{-1} \mathrm{MHz}^{-1}$. The values in brackets are the averaged percentages of the actual electric field values.

and the background noise:

$\Delta \epsilon=\sqrt{\left(\frac{\delta \epsilon}{\delta t}\right)^{2}+\left(\frac{\delta \epsilon}{\delta \alpha}\right)^{2}+\left(\frac{\delta \epsilon}{\delta \phi}\right)^{2}}$.

Here, $\left(\frac{\delta \epsilon}{\delta t}\right)$ is the statistical uncertainty (RMS) of the background noise (see Sect. 4.7); $\left(\frac{\delta \epsilon}{\delta \alpha}\right)$ is the statistical uncertainty of the E-field dependence on the beam-forming direction; and $\left(\frac{\delta \epsilon}{\delta \phi}\right)$ is an estimated uncertainty of $5 \%$ introduced by phase errors remaining after delay calibration (Horneffer 2006).

The dependence of the E-field on the beam-forming direction is implemented in the gain calibration and a statistical uncertainty is estimated for each direction and frequency sub-band calculating a gradient $\frac{\delta G}{\delta \alpha}$ on the beampattern:

$$
\begin{aligned}
\epsilon & =k \cdot \frac{1}{\sqrt{G(\alpha)}} \\
\left(\frac{\delta \epsilon}{\delta \alpha}\right) & =k \cdot-\frac{1}{2} G(\alpha)^{-1.5} \cdot \frac{\delta G}{\delta \alpha} \\
& =k \cdot \frac{1}{\sqrt{G(\alpha)}} \cdot-\frac{1}{2 G(\alpha)} \cdot \frac{\delta G}{\delta \alpha} .
\end{aligned}
$$

Here, $\epsilon$ is the electric field strength, $k$ is a constant factor (see Eq: 1), $G(\alpha)$ is the direction dependent gain factor, and $\frac{\delta G}{\delta \alpha}$ is its gradient.

The determined uncertainties are consistent within the uncertainties applicable to earlier LOPES results Horneffer 2006). For all 23 analyzed events, the average uncertainties are listed in Table 1

\section{Results}

For the electric field spectra, a frequency resolution of $2.5 \mathrm{MHz}$ (16 bins over the $40 \mathrm{MHz}$ band) was chosen as a compromise between pulse broadening and sufficient spectral resolution to reveal phase uncertainties. The phase uncertainties are mainly caused by narrowband RFI and deviations from the frequency dependent gain calibration. The radio spectra from Monte Carlo simulations were parametrized with an exponential (Huege \& Falcke 2005b). For comparison, an exponential $\epsilon_{\nu}=K \cdot \exp (\nu / \mathrm{MHz} / \beta)$ and a power-law form $\epsilon_{\nu}=K \cdot \nu^{\alpha}$ were fitted to the data of the single and the average spectra obtained by both methods and corrected as described in Sect. 4.7. However, the $\chi_{\text {red }}^{2}$ of both fit types turns out to be the same and thus both functions fit equally well. The resulting fit values can be found in Sect. 5.2 and Table 2 at the end of the paper. 


\subsection{Single event spectra}

Radio spectra were calculated for 23 LOPES events. Characteristic values for each event are listed in Table 2 including the event number; the event date; the electric field strength measured in the whole LOPES band from the spectra $\epsilon$; the spectral index $\alpha$; the exponential fit parameter $\beta$; the width of the Gaussfitted full-band pulse $\Delta t$; the E-field peak SNR from the spectra in sigma $\sigma$; the estimated primary energy $E_{p}$ (http://www-ik.fzk.de/ ${ }^{\sim}$ ralph/CREAM1.php Glasstetter et al. 2005, only reliable down to $48^{\circ}$ elevation, no values are given below $20^{\circ}$ elevation); the direction (azimuth $\phi$ and elevation $\theta$ ); the angle of the shower axis with the geomagnetic field $\vartheta$; the average distance of the antennae to the shower core $d$; and the number of antennae used for beam-forming $N$. The values for $\epsilon, \alpha, \beta, \Delta t$, and $\sigma$ are given as the average result from the cc-beam and the f-beam. The error was chosen as the maximum from both methods.

Additionally, six single event spectra for both methods are plotted in Fig. 14. For each spectrum an average noise spectrum was calculated by FFT of 50 blocks offset from the cosmic-ray radio pulse, which is plotted as well. Furthermore, two spectra from Monte Carlo simulations are added to each plot for an inclined shower with $\theta=45^{\circ}$ and for a vertical shower with $\theta=90^{\circ}$ (Huege et al. 2007). The showers were simulated for a proton with a primary energy of $E_{p}=10^{17} \mathrm{eV}$, an azimuthal viewing angle of $\phi=45^{\circ}$ from the observer measured at a shower core distance of $100 \mathrm{~m}$ and were scaled to have the same average as the single spectra for comparison.

The values on the top of the plots in Fig. 14 read; in the 1st line: the event number, the measured electric field strength from the spectra, the E-field peak SNR from the spectra in sigma, the estimated primary energy (Glasstetter et al. 2005), the width of the Gauss-fitted fullband pulse, the direction (azimuth angle and elevation angle) and the geomagnetic field angle; in the 2nd line: the fit parameters $\alpha$ and $\beta$ for the spectral slope. All the E-field values, slope values, sigma values, and width values in the table and the plots in the appendix are given as the average of the f-beam and the cc-beam method together with the maximum uncertainty.

The six plotted events have the following special characteristics. The analyzed events indicate that with larger pulse width, lower frequencies dominate and steepen the spectrum (see event [16] with a width of $51 \mathrm{~ns}$ and a flat spectrum, versus event [7] with a width of $101 \mathrm{~ns}$ and a steep spectrum). The latter event was found to be recorded during thunderstorm activity in the vicinity of LOPES. Strong electric fields in the clouds may have enhanced the radio emission and broadened the pulse (Buitink et al. 2007). However, the sample is too small and no significant dependence was found. Furthermore, the measured electric field amplitude increases with increasing angle of the shower axis with the geomagnetic field, as expected, according to the geomagnetic emission mechanism (compare event [10] with $11 \mu \mathrm{V} \mathrm{m}^{-1} \mathrm{MHz}^{-1}$ and $51^{\circ}$, versus event [5] with $20 \mu \mathrm{V} \mathrm{m}^{-1} \mathrm{MHz}^{-1}$ and $\left.89^{\circ}\right)$. In addition, two events with the largest electric field amplitude are plotted in Fig. 14, which have the smallest uncertainties (see event [20] and [21] with geomagnetic angles of $78^{\circ}$ and $70^{\circ}$ ).

\subsection{Average field spectrum}

An average field spectrum was calculated to obtain an average spectral slope for the 23 selected LOPES events (Fig. 12). For this plot, the single event spectra were normalized by their mean to unity to make them energy independent and then they were averaged. The numbers on the top indicate the number of events taken into account for the average spectrum and the parameters from the spectral fits to the plotted spectra. The average exponential fit with the parameter $\beta$ is plotted for the two methods.

The exponential parameter is obtained with $\beta=$ $-0.017 \pm 0.004$ and the spectral index is obtained with $\alpha=-1.0 \pm 0.2$. These fit parameters apply to an average zenith angle of the 23 events of $53^{\circ}$ and an average distance of the antennae from the shower core position of $76 \mathrm{~m}$.

The average spectrum measured confirms basic expectations of Falcke \& Gorham (2003), but it is not consistent within the uncertainties and it is slightly steeper than the slope obtained from Monte Carlo simulations based on air showers simulated with CORSIKA (Cosmic Ray Simulations for KASCADE). The fit parameters from simulations of a $45^{\circ}$ inclined air shower are $\beta=-0.0085$ and $\alpha=-0.49$; and the values for a vertical shower are $\beta=-0.012$ and $\alpha=-0.7$ (Huege et al. 2007). For a closer comparison, simulations on single event basis are necessary taking into account polarization characteristics of the LOPES antennae and those simulations have not yet been performed.

For further comparison, the spectral index recently measured by the CODALEMA group for a single event spectrum is $\alpha=-1.5 \pm 0.2$ (Ardouin et al. 2006), which is consistent with the average slope we obtained for the uncorrected spectra.

\subsection{Discussion of methods}

The major difference in the determination of the electric field strength, between the calculation of the spectra and the standard analysis software, is that the latter fits a Gaussian to the pulse. The standard software assumes the pulse to be Gaussian and any deviation of the real cosmicray radio pulse shape will cause deviations in the resulting electric field strength. Thus, the method used here for the spectra is more sensitive to the shape of the radio pulses. The electric field strengths determined from the spectra agree within $\pm 30 \%$ with those obtained with the standard analysis (one sigma statistical uncertainty).

The two methods for the spectra determination are in statistical agreement. The noise on the cc-beam is a few percent $(\sim 3 \%)$ larger than the noise on the f-beam (see Sect. 4.7). Furthermore, the f-beam method is more accurate, since the statistical uncertainties of all events averaged by weighting is only half of the value obtained for the cc-beam method. Additionally, the $\chi^{2}$ of the fits on the spectra from the f-beam method are on average an order of magnitude better than for the cc-beam method.

\subsection{Pulse width analysis}

The resulting average radio spectrum can be used to obtain an average shape for the measured radio pulses by inverse Fourier transformation. However, the phases are modified 
by the electronics, which at the moment, we cannot correct for. Thus, a lower limit on the pulse width can be provided by applying an inverse Fourier transformation on the measured spectral amplitudes (see Fig. 13). The obtained pulse was upsampled in frequency to increase the number of samples for a fit. The amplitude was normalized to unity, since it is not comparable with earlier E-field values. A Gaussian fit on the obtained pulse results in a full-width-half-maximum (FWHM) of 40 ns. As expected, this minimum is slightly smaller than the impulse response of the electronics of $\sim 57 \mathrm{~ns}$. The average pulse width of the analyzed events resulted in $60 \pm 20 \mathrm{~ns}$ (one sigma statistical uncertainty). This value was calculated on 41 events, for which the difference between the f-beam width and the cc-beam width was not larger than two samples (25 ns). The two samples are a quality criterion. The simulated and measured pulse width set an upper limit of $60 \mathrm{~ns}$ and the minimum pulse width a lower limit of 40 ns for the single selected LOPES pulses. Therefore, the obtained spectral slopes provide an upper limit, possibly broadened by unknown phase uncertainties, which were estimated for the uncertainties in the spectra.

\section{Conclusion and outlook}

With a sample of 23 strong LOPES events, we measured the radio spectrum received from cosmic-ray air showers detected with up to 24 simple dipole antennae oriented in the East-West direction over a frequency band of $40 \mathrm{MHz}$. The spectra show that a direct Fourier transformation can be performed on the beam-formed radio pulses measured with LOFAR. The accuracy with which the spectral amplitudes can be obtained is limited by the instrument noise and phase uncertainties. Furthermore, the quality of the spectral slope is limited by the quality of the antenna gain model, which was simulated and measured in several calibration campaigns.

The average slope of the spectra obtained with LOPES confirms basic expectations, but it is not consistent within uncertainties and it is slightly steeper than the slope obtained from Monte Carlo simulations based on air showers simulated with CORSIKA. The simulations show for the LOPES band flatter spectra to higher zenith angles and larger distances from the shower core position, and they show steeper spectra for increasing the azimuth from direction North to direction East.

As expected, the spectral slopes of the selected sample of events depend on the length of the pulse, where longer pulses result in steeper spectra. However, taking into account the low number statistics, the spectra do not show a significant dependence of the slope on the electric field amplitude, the azimuth angle, the zenith angle, the curvature radius, nor on the average distance of the antennae from the shower core position.

According to the obtained spectral slopes, the maximum power is emitted below $40 \mathrm{MHz}$. Furthermore, the decrease in power to higher frequencies indicates a loss in coherence determined by the shower disc geometry and the longitudinal distribution of particles therein. In Monte Carlo simulations, the latter was found to follow a broadened gammadistribution having a long tail to several meters in shower thickness, which confirms a smooth loss of coherence to higher frequencies (see Fig. 7 in Huege et al. 2007).
The selected sample of events contained one event that occurred during thunderstorm activity in the vicinity of LOPES. This thunderstorm event had a large pulse width and a very steep spectrum, suggesting that LOPES measured a geoelectric effect for the first time.

For the study of the lateral dependence of the air shower radio spectrum, as a function of distance to the shower axis, a larger array with longer baselines and more sensitive antennae is needed. LOFAR will have a very dense core with baselines up to 500 meters, a collecting area of $\sim 10000 \mathrm{~m}^{2}$ at $75 \mathrm{MHz}$, and a frequency range from $10 \mathrm{MHz}$ to $290 \mathrm{MHz}$ sampled at $200 \mathrm{MHz}$, and thus will be perfectly suited to improve on results obtained here. Most importantly, we will probe the coherence of the air shower radio signal to higher frequencies to infer on the geometry of the shower disc. Understanding the shower geometry will allow us to determine the primary particle species using radio only.

Furthermore, within the Pierre Auger collaboration, a sparse radio antenna array is under development van den Berg \& The Pierre Auger Collaboration 2007). The antennae will be tested in the field of the Pierre Auger Observatory (PAO, www . auger . org). The PAO measures cosmic-ray air showers with particle detectors in an area of 3000 square kilometers with distances between the particle detector stations of $1.5 \mathrm{~km}$. Therefore, the PAO responds to a higher primary particle energy range than LOPES, which lies beyond $10^{18} \mathrm{eV}$.

A next step will be the simulation of complete air showers based on parameters provided by KASCADE and LOPES, including the hardware response of LOPES. Such simulations will allow a close comparison of theory and experiment.

Acknowledgments. Andreas Nigl gratefully acknowledges a grant from ASTRON, which made this work possible. LOPES was supported by the German Federal Ministry of Education and Research. The KASCADE-Grande experiment is supported by the German Federal Ministry of Education and Research, the MIUR and INAF of Italy, the Polish Ministry of Science and Higher Education and the Romanian Ministry of Education and Research.

\section{References}

Allan, H. R. 1971, Prog. in Element. part. and Cos. Ray Phys., Vol. 10,171

Allan, H. R., Clay, R. W., \& Jones. 1970, Nature, 225, 253

Allan, H. R., Clay, R. W., Jones, J. K., Abrosimov, A. T., \& Neat, K. P. 1969, Nature, 222, 635

Allan, H. R. \& Jones, J. K. 1966, Nature, 212, 129

Allan, H. R., Neat, K. P., \& Jones, J. K. 1967, Nature, 215, 267

Antoni, T., Apel, W. D., Badea, F., et al. 2003, Nuclear Instruments and Methods in Physics Research Section A, 513, 490

Ardouin, D., Bellétoile, A., Charrier, D., et al. 2006, Astroparticle Physics, 26, 341

Arts, M. 2005, EM simulations of a LOFAR LBH antenna, personal communication

Askaryan, G. A. 1962, JETP, 14, 441

Auger Collaboration: J. Abraham et al. 2007, Sci, 318, 938

Buitink, S., Apel, W., Asch, T., et al. 2007, A\&A

Falcke, H., Apel, W. D., Badea, A. F., et al. 2005, Nature, 435, 313

Falcke, H. \& Gorham, P. 2003, Astroparticle Physics, 19, 477

Glasstetter, R., Antoni, T., Apel, W. D., et al. 2005, in Proc. Int. Cosmic Ray Conf. (ICRC), 2005, 293

Gurevich, A. V., Karashtin, A. N., Chubenko, A. P., et al. 2004, ApJ, 325,389

Horneffer, A. 2006, Phd-thesis, Universität Bonn

Horneffer, A., Apel, W. D., Arteaga, J. C., et al. 2007, in Proc. Int. Cosmic Ray Conf. (ICRC), 2007 
Horneffer, A., Apel, W. D., Badea, A. F., et al. 2004, in Gravitational Wave and Particle Astrophysics Detectors, Proc. SPIE, Vol. 5500, 2004, ed. J. Hough \& G. H. Sanders, 129-138

Huege, T. \& Falcke, H. 2005a, A\&A, 430, 779

Huege, T. \& Falcke, H. 2005b, Astroparticle Physics, 24, 116

Huege, T., Ulrich, R., \& Engel, R. 2007, Astroparticle Physics

Jelley, J. V., Fruin, J. H., Porter, N. A., et al. 1965, Nature, 205, 327

Kahn, F. D. \& Lerche, I. 1966, Proc. Royal Society of London Series A, 289, 206

Mandolesi, N., Morigi, G., \& Palumbo, G. G. C. 1974, Journal of Atmospheric and Terrestrial Physics, 36, 1431

Navarra, G., Antoni, T., Apel, W. D., et al. 2004, Nuclear Instruments and Methods in Physics Research A, 518, 207

Nehls, S., Hakenjos, A., Arts, M. J., et al. 2007, subm., NIM A

Prah, J. H. 1971, M.Phil. thesis, University of London

Spencer, R. E. 1969, Nature, 222, 460

Sun, M. P. 1975, PhD thesis, University of London

van den Berg, A. M. \& The Pierre Auger Collaboration. 2007, in Proc. Int. Cosmic Ray Conf. (ICRC), 2007

1 Department of Astrophysics, IMAPP, Radboud University Nijmegen, P.O. Box 9010, 6500 GL Nijmegen, The Netherlands

e-mail: anigl@astro.ru.nl

2 Institut für Kernphysik, Forschungszentrum Karlsruhe, 76021 Karlsruhe, Germany

3 Institut für Experimentelle Kernphysik, Universität Karlsruhe (TH), 76021 Karlsruhe, Germany

4 Institut für Prozessverarb. und Elektr., Forschungszentrum Karlsruhe, 76021 Karlsruhe, Germany

5 Fachbereich Physik, Universität Wuppertal, 42097 Wuppertal, Germany

6 ASTRON, 7990 AA Dwingeloo, The Netherlands

7 Dipartimento di Fisica Generale dell'Università, 10125 Torino, Italy

8 Max-Planck-Institut für Radioastronomie, 53010 Bonn, Germany

9 National Institute of Physics and Nuclear Engineering, 7690 Bucharest, Romania

10 Fachbereich Physik, Universität Siegen, 57068 Siegen, Germany

11 Istituto di Fisica dello Spazio Interplanetario, INAF, 10133 Torino, Italy

12 Soltan Institute for Nuclear Studies, 90950 Lodz, Poland

13 Physics Department, Bucharest University, BucharestMagurele, P.O. Box MG-11, RO-077125, Romania
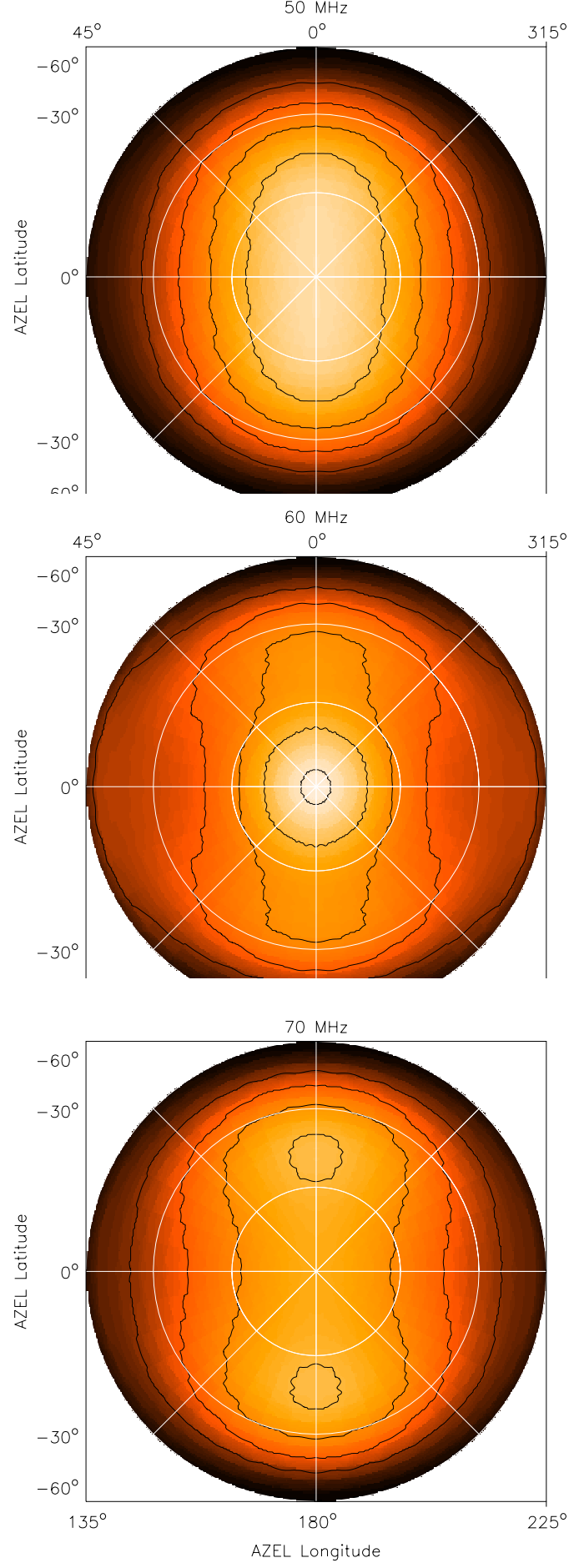

Figure 3. Simulated gain patterns for a single LOPES dipole antenna, for all directions in azimuth $\phi$ and elevation $\theta$, at frequencies $50 \mathrm{MHz}$ (top), $60 \mathrm{MHz}$ (middle) and $70 \mathrm{MHz}$ (bottom), for a bandwidth of $1 \mathrm{MHz}$. The zenith lies in the center of each plot and the white circles increase in steps of $30^{\circ}$ in elevation down to the horizon at the plot edges. The antenna dipole is lying horizontally in the plots. The gain ranges from its minimum in black (gain $=0$ ) to the maximum gain in white (gain $=5$ ). The dark contours are plotted in steps of $20 \%$. Where five contours are visible (middle plot), they go from $20 \%$ gain to $100 \%$ gain. 


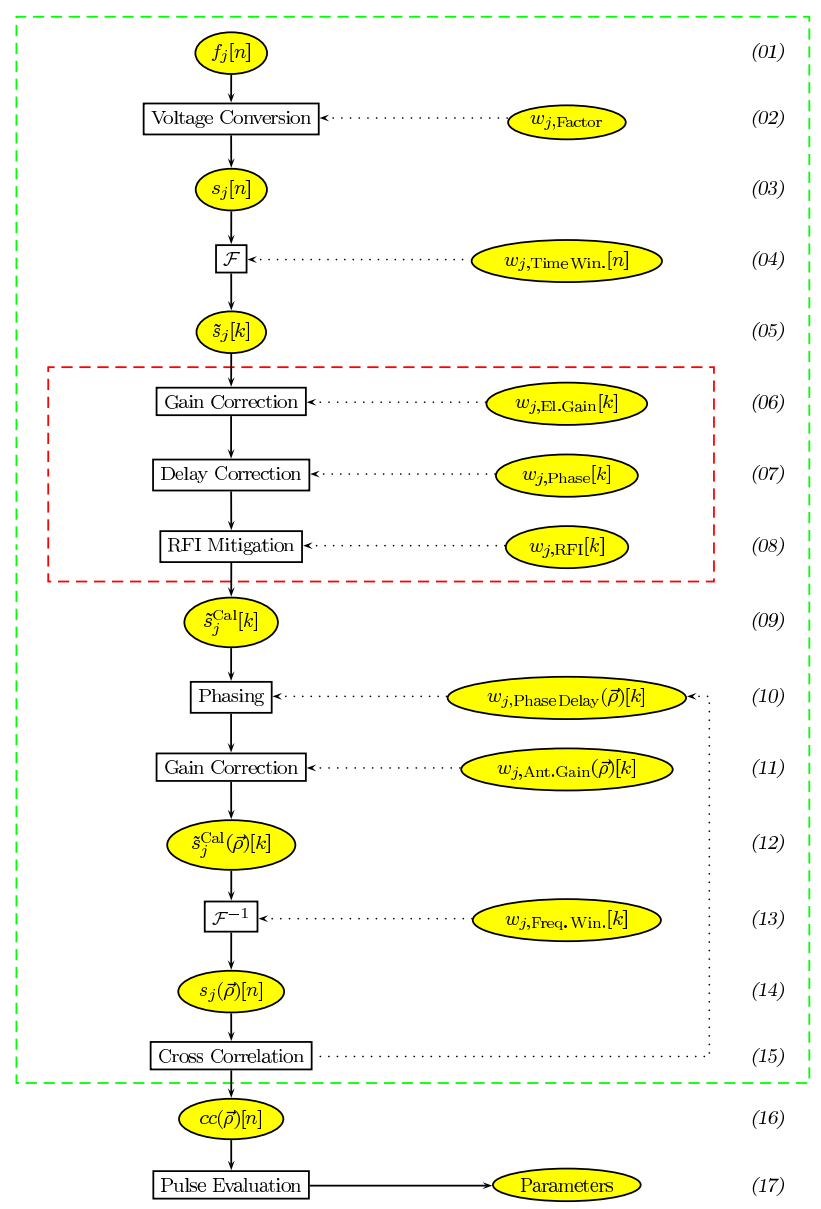

Figure 4. This flowchart of the cosmic-ray processing pipeline starts from the raw data $f_{j}[n]$, where $n$ is the index for the ADC samples per antenna $j$. The signal converted from ADC counts to voltage is denoted as $s$. The Fourier transformation is denoted as $\mathcal{F}$ and the inverse Fourier transformation as $\mathcal{F}^{-1}$. The number of the frequency bins after Fourier transformation is indicated with $k$. Directional dependence of the signal and the weights is indicated by the vector $\vec{\rho}$. The outer dashed line encloses the processing per individual dipole and the inner dashed line encloses the signal calibration steps in the frequency domain. A more detailed description of the steps in the processing pipeline can be found in Sect. 4.1 .

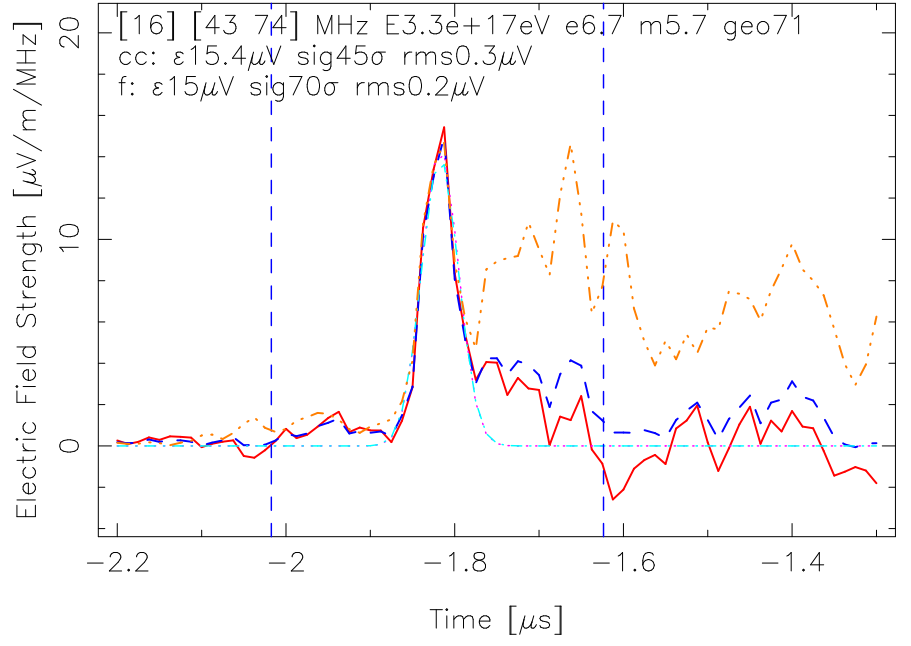

Figure 5. Comparison of full-band cross-correlation beam (cc-beam, solid line), full-band field strength beam (f-beam, dashed), and full-band power beam (p-beam, dash dotted) for a characteristic strong event. Additionally, Gauss-fits to the cc-beam and f-beam are plotted (thin dashed line and thin dotted line). The vertical lines (dashed) indicate the time window of the f-beam for which the FFT was applied.

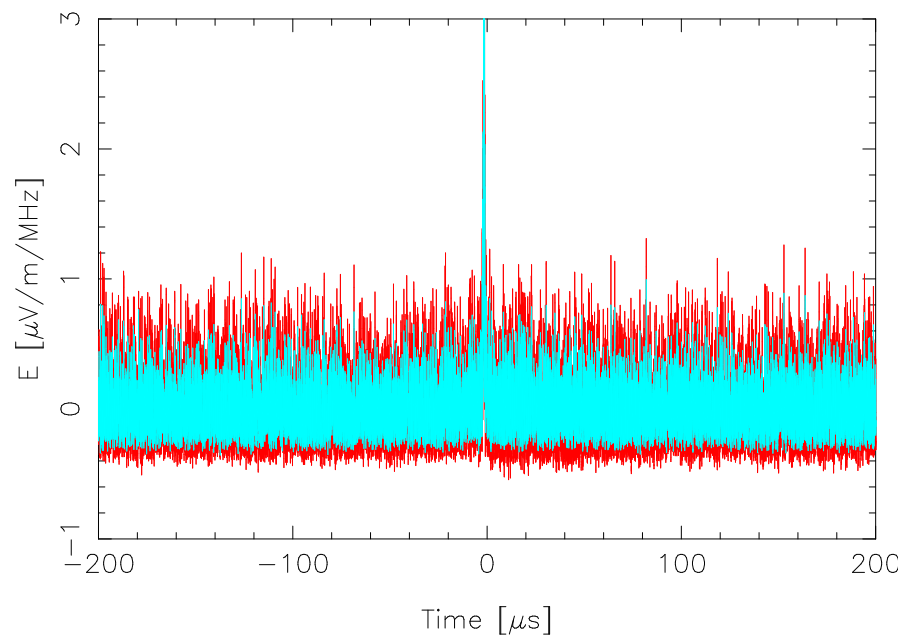

Figure 6. Comparison of the noise of the cross-correlation beam (cc-beam: large RMS) and the noise of the field strength beam (f-beam: small RMS) of a LOPES event for the period of $0.2 \mathrm{~ms}$ before and after the trigger. The peak slightly left of center of the time-window is the air shower pulse. 


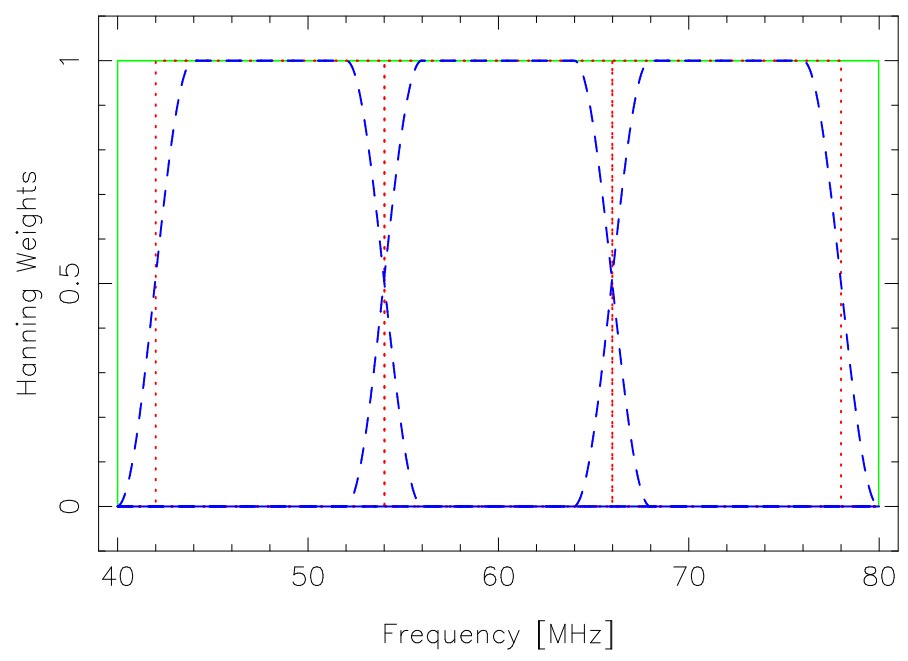

Figure 7. Modified Hanning windows (dashed) and rectangular windows (dotted) for three sub-bands of the frequency band from $40 \mathrm{MHz}$ to $80 \mathrm{MHz}$.

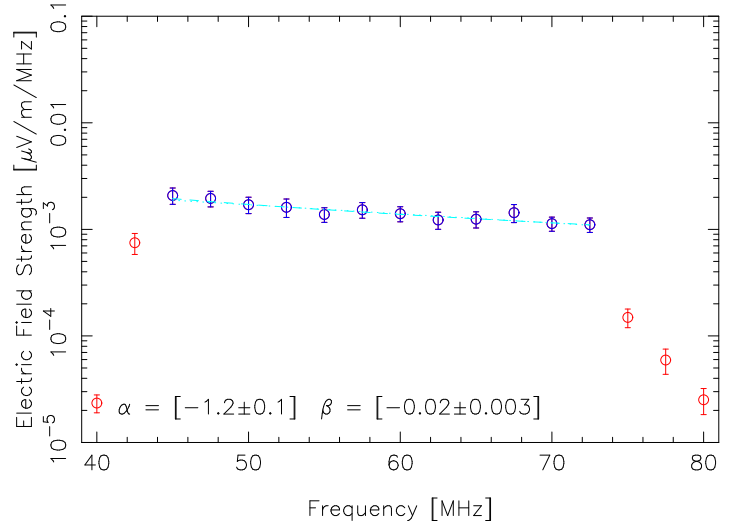

Figure 8. System noise spectrum of the LOPES beam to the zenith in units of electric field strength calculated on 664 events of $1 \mathrm{~ms}$ recorded over a period of one year $(11 / 2005-11 / 2006)$. The numbers on the bottom of the plot are, respectively, the slope parameters $\alpha$ and $\beta$ for a power-law function and an exponential function fitted to the spectrum (dashed line).

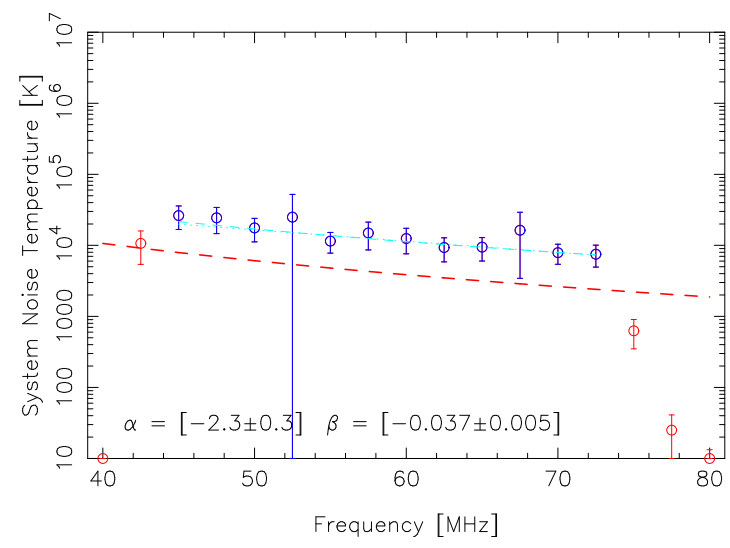

Figure 9. Spectrum of the LOPES beam to the zenith in units of noise temperature calculated on 664 events of $1 \mathrm{~ms}$ recorded over a period of one year. The numbers on the bottom of the plot are the slope parameters $\alpha$ and $\beta$ for a power-law function and an exponential function fitted to the spectrum (dashed line). The galactic noise is plotted for comparison (lower dashed line). 


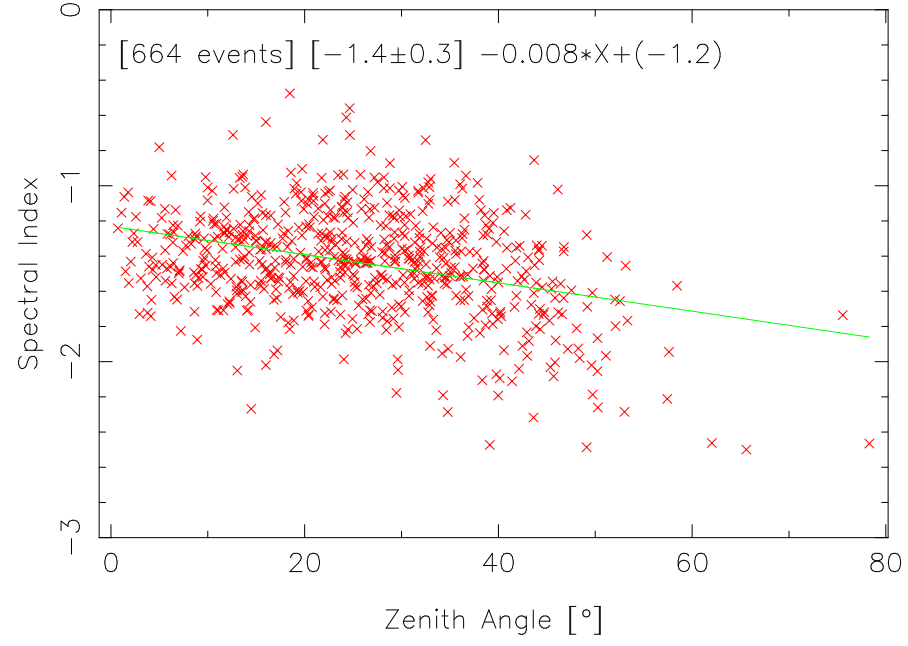

Figure 10. Spectral index of 664 noise spectra as a function of zenith angle. The average slope is $\alpha=-1.4 \pm 0.3$ and the fitted line is described by $-0.008 \times\left(90-\theta /{ }^{\circ}\right)-1.2$.

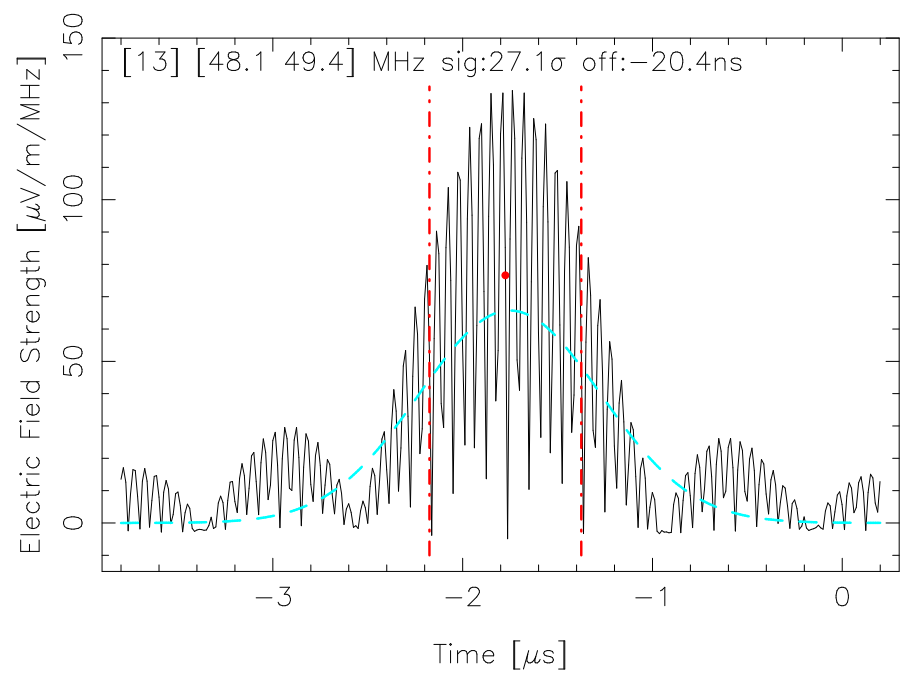

Figure 11. Cross-correlation beam filtered in a frequency sub-band (thin solid line) and scaled full-band Gauss-fit (dashed). The vertical lines (dash-dotted) indicate the beginning and end of the part of the cc-beam that was averaged and the dot in the middle indicates the resulting value of $76.6 \mu \mathrm{V} \mathrm{m}^{-1} \mathrm{MHz}^{-1}$.

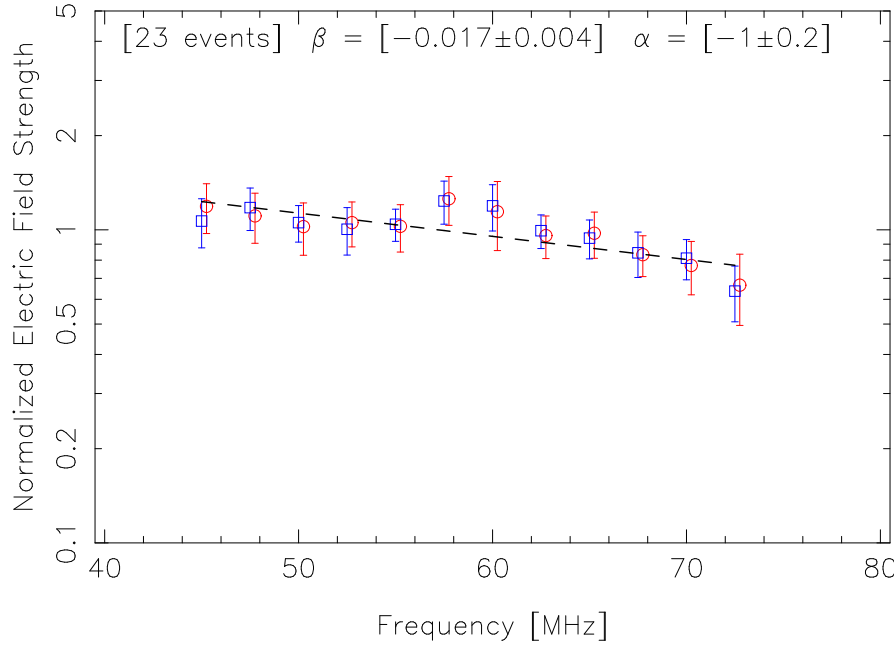

Figure 12. Comparison of average cosmic-ray electric field spectra obtained with 23 LOPES events by two different methods. Before averaging, the single spectra are normalized to unity. The frequency bin values are determined on the cc-beam (circles) and the Fourier transformation of the f-beam (squares) are fitted with one exponential, visible as a straight line in this log-linear plot (dashed line).

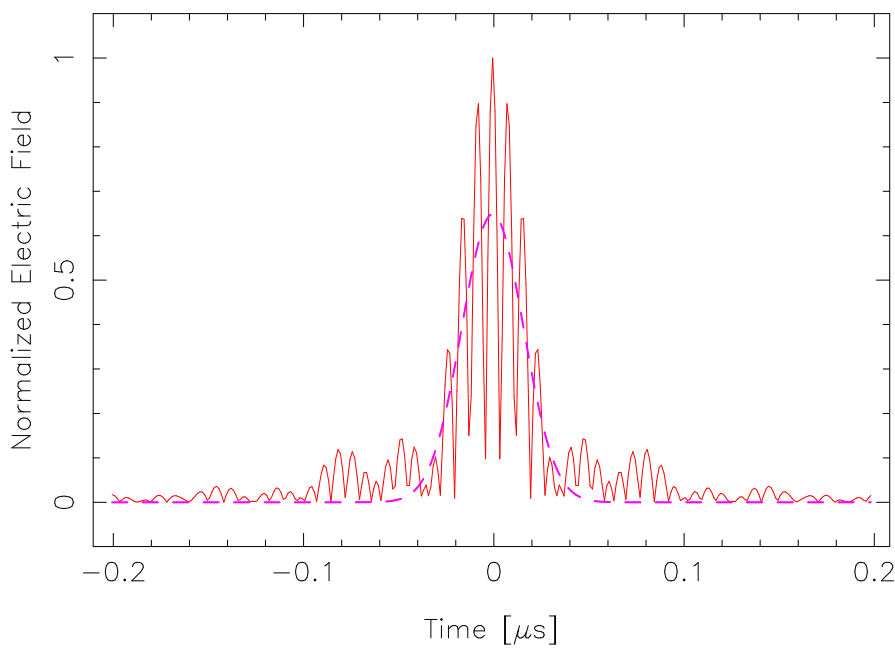

Figure 13. Pulse obtained by inverse Fourier transformation of the amplitudes of the average electric field spectrum (solid line). The width of the Gaussian fit results in $40 \mathrm{~ns}$ (dashed line). The amplitude is normalized to unity. 
Table 2. Event parameter list for all 23 analyzed events. For more details see first paragraph of Sect. 5.1

\begin{tabular}{|c|c|c|c|c|c|c|c|c|c|c|c|}
\hline$\#$ & Date (Filename) & E-field & Index & Expo. & Width & Sig. & Energy & AZEL & Geom. Ang. & Dist. & Ant. \\
\hline & Symbol & $\epsilon$ & $\alpha$ & $\beta$ & $\Delta t$ & $\sigma$ & $E_{p}$ & $\phi-\theta$ & $\vartheta$ & $d$ & $N$ \\
\hline & Unit & $\mu \mathrm{V} / \mathrm{m} / \mathrm{MHz}$ & - & - & ns & - & eV] & $10]$ & T & {$[\mathrm{m}$} & - \\
\hline 1 & 2006.03.14.13:30:27 & $\begin{array}{l}11 \pm 4 \\
\end{array}$ & $-1.8 \pm 0.5$ & $-0.031 \pm 0.008$ & $\overline{50}$ & 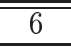 & $\begin{array}{c}(\ldots) \\
\end{array}$ & $0-20$ & $\overline{95}$ & $\overline{70 \pm 50}$ & 24 \\
\hline 2 & 2006.03.23.04:01:07 & $17 \pm 4$ & $-1.1 \pm 0.4$ & $-0.019 \pm 0.007$ & 77 & 13 & $3.60 \mathrm{E}+17$ & $35-51$ & 60 & $80 \pm 40$ & 23 \\
\hline 3 & $2006.04 .05 .05: 39: 13$ & $25 \pm 5$ & $-1.1 \pm 0.8$ & $-0.02 \pm 0.01$ & 49 & 16 & $5.40 \mathrm{E}+17$ & $49-47$ & 61 & $70 \pm 30$ & 23 \\
\hline 4 & $2006.05 .01 .10: 31: 40$ & $19 \pm 4$ & $-1.9 \pm 0.4$ & $-0.031 \pm 0.006$ & 80 & 14 & $1.50 \mathrm{E}+17$ & $286-37$ & 64 & $80 \pm 30$ & 23 \\
\hline 5 & 2006.06.14.11:03:15 & $20 \pm 6$ & $-0.4 \pm 0.3$ & $-0.008 \pm 0.005$ & 57 & 46 & $1.00 \mathrm{E}+18$ & $340-24$ & 89 & $80 \pm 50$ & 14 \\
\hline 6 & $2006.06 .19 .19: 21: 24$ & $6 \pm 3$ & $-0.8 \pm 0.3$ & $-0.013 \pm 0.004$ & 47 & 5 & $1.80 \mathrm{E}+17$ & $12-34$ & 79 & $80 \pm 40$ & 20 \\
\hline 7 & 2006.08.19.23:01:41 & $25 \pm 6$ & $-3.6 \pm 0.4$ & $-0.061 \pm 0.006$ & 101 & 60 & $2.50 \mathrm{E}+17$ & $20-41$ & 73 & $70 \pm 40$ & 9 \\
\hline 8 & $2006.09 .11 .05: 24: 22$ & $15 \pm 5$ & $-1.2 \pm 0.4$ & $-0.021 \pm 0.006$ & 77 & 8 & $1.50 \mathrm{E}+17$ & $341-38$ & 75 & $80 \pm 40$ & 9 \\
\hline 9 & $2006.09 .22 .17: 58: 45$ & $19 \pm 6$ & $-0.8 \pm 0.6$ & $-0.01 \pm 0.01$ & 51 & 8 & $4.90 \mathrm{E}+17$ & $56-50$ & 57 & $90 \pm 40$ & 8 \\
\hline 10 & $2006.10 .13 .10: 43: 03$ & $11 \pm 4$ & $-1.9 \pm 0.8$ & $-0.03 \pm 0.01$ & 85 & 35 & $1.90 \mathrm{E}+17$ & $0-64$ & 51 & $90 \pm 50$ & 9 \\
\hline 11 & $2006.11 .02 .07: 14: 52$ & $24 \pm 7$ & $-2 \pm 0.4$ & $-0.034 \pm 0.006$ & 67 & 9 & $2.20 \mathrm{E}+17$ & $328-30$ & 81 & $70 \pm 40$ & 9 \\
\hline 12 & $2005.12 .05 .06: 46: 13$ & $29 \pm 7$ & $-0.8 \pm 0.3$ & $-0.014 \pm 0.004$ & 55 & 13 & $3.10 \mathrm{E}+18$ & $315-22$ & 87 & $60 \pm 30$ & 16 \\
\hline 13 & $2005.12 .08 .09: 10: 45$ & $31 \pm 9$ & $-0.4 \pm 0.6$ & $-0.005 \pm 0.01$ & 113 & 10 & $(\ldots)$ & $75-15$ & 82 & $60 \pm 30$ & 16 \\
\hline 14 & $2006.01 .18 .13: 46: 06$ & $11 \pm 3$ & $-1.2 \pm 0.5$ & $-0.022 \pm 0.008$ & 59 & 8 & $2.00 \mathrm{E}+17$ & $357-49$ & 66 & $70 \pm 30$ & 18 \\
\hline 15 & $2006.01 .19 .06: 26: 13$ & $11 \pm 3$ & $-1.3 \pm 0.7$ & $-0.02 \pm 0.01$ & 52 & 8 & $1.30 \mathrm{E}+17$ & $347-43$ & 71 & $80 \pm 40$ & 18 \\
\hline 16 & $2006.02 .04 .13: 33: 28$ & $14 \pm 4$ & $-0.1 \pm 0.4$ & $-0.002 \pm 0.007$ & 51 & 57 & $3.30 \mathrm{E}+17$ & $4-44$ & 71 & $70 \pm 40$ & 18 \\
\hline 17 & $2006.02 .07 .09: 40: 20$ & $27 \pm 7$ & $-1.7 \pm 0.3$ & $-0.029 \pm 0.005$ & 49 & 6 & $2.90 \mathrm{E}+17$ & $325-30$ & 81 & $100 \pm 50$ & 21 \\
\hline 18 & $2006.02 .10 .08: 11: 20$ & $14 \pm 4$ & $-0.8 \pm 0.4$ & $-0.014 \pm 0.006$ & 52 & 13 & $1.60 \mathrm{E}+17$ & $345-36$ & 77 & $80 \pm 30$ & 24 \\
\hline 19 & $2006.02 .14 .02: 09: 17$ & $40 \pm 10$ & $-1.2 \pm 0.3$ & $-0.02 \pm 0.005$ & 56 & 9 & $(\ldots)$ & $28-12$ & 101 & $60 \pm 40$ & 16 \\
\hline 20 & 2006.02.18.19:30:41 & $66 \pm 10$ & $-1 \pm 0.3$ & $-0.018 \pm 0.005$ & 48 & 292 & $3.60 \mathrm{E}+17$ & $9-37$ & 78 & $80 \pm 40$ & 24 \\
\hline 21 & $2006.02 .20 .12: 26: 29$ & $40 \pm 8$ & $-1.1 \pm 0.4$ & $-0.019 \pm 0.006$ & 98 & 88 & $7.70 \mathrm{E}+17$ & $278-26$ & 70 & $60 \pm 40$ & 24 \\
\hline 22 & $2006.02 .23 .03: 34: 39$ & $9 \pm 3$ & $-0.8 \pm 0.5$ & $-0.014 \pm 0.008$ & 57 & 7 & $2.10 \mathrm{E}+17$ & $322-43$ & 68 & $90 \pm 40$ & 24 \\
\hline 23 & $2006.03 .07 .16: 55: 47$ & $11 \pm 3$ & $-1.4 \pm 0.7$ & $-0.02 \pm 0.01$ & 124 & 8 & $2.60 \mathrm{E}+17$ & $4-65$ & 49 & $100 \pm 50$ & 23 \\
\hline
\end{tabular}


Figure 14. Single event cosmic-ray electric field spectra determined on six LOPES example events. Each plot includes a spectrum determined on the cc-beam (circles) for 12 sub-bands, a spectrum determined on the f-beam (squares) by FFT of 32 time-samples around the radio pulse, and a noise spectrum determined by FFT on 50 blocks of 32 samples offset from the pulse (squares in the middle of each plot). The spectra of the cosmic-ray air shower radio pulse are fitted with an exponential, visible as a straight line in these log-linear plots (cc-beam: dash-dotted \& f-beam: dash-dot-dot). In addition, the simulated data of an inclined shower (dotted) and a vertical shower (dashed) are plotted. For more details see the second to last paragraph of Sect. 5.1
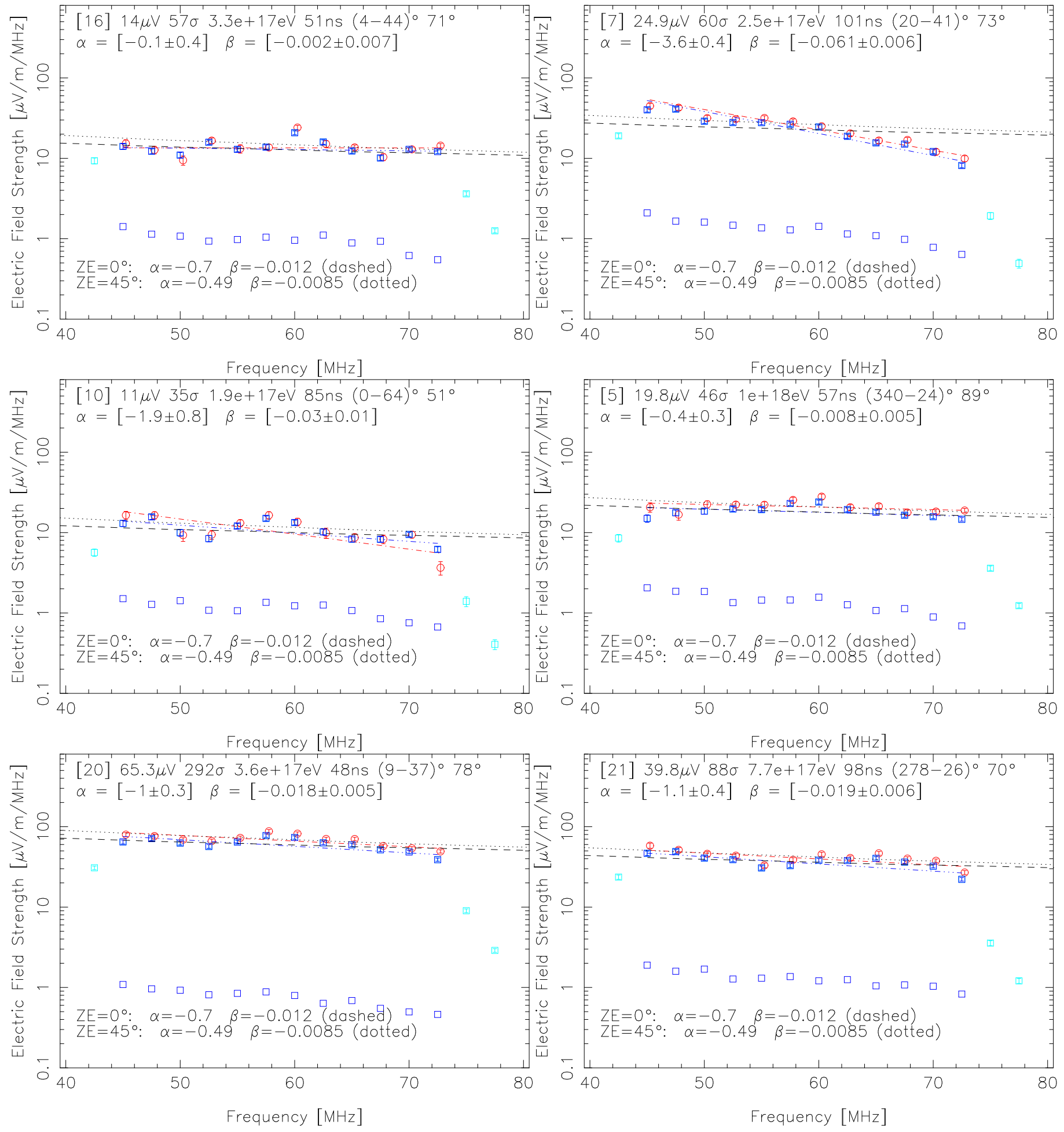